\title{
Interactive Paper Substrates to Support Musical Creation
}

\author{
Jérémie Garcia ${ }^{1,2}$ \\ garcia@lri.fr \\ ${ }^{1}$ INRIA \\ Univ Paris-Sud \& CRNS (LRI) \\ F-91405 Orsay, France \\ Theophanis Tsandilas ${ }^{1}$ \\ fanis@1ri.fr \\ Carlos Agon ${ }^{2}$ \\ agon@ircam.fr
${ }^{2}$ IRCAM, CNRS UMR STMS
1 place Igor Stravinsky
F-75004 Paris, France

\author{
Wendy E. Mackay ${ }^{1,3}$ \\ mackay@1ri.fr
}

\begin{abstract}
We present paper substrates, interactive paper components that support the creation and manipulation of complex musical data. Substrates take different forms, from whole pages to movable strips, and contain or control typed data representations. We conducted participatory design sessions with five professional musicians with extensive experience with music creation tools. All generated innovative uses of paper substrates, manipulating their data, linking multiple representation layers and creating modular, reusable paper elements. The substrates reflect the structure of their computer-based data, but in a much more flexible and adaptable form. We use their prototypes to provide concrete examples of substrates, identify their roles, properties and functions. Finally, we explore their physical and interaction design with an interactive prototype.
\end{abstract}

\section{AUTHOR KEYWORDS}

Interactive paper; tangible user interfaces; musical creation; computer-aided composition; participatory design.

\section{ACM Classification Keywords}

H.5.2 Information Interfaces and Presentation: User Interfaces-User-centered design, Input devices and strategies.

\section{General Terms}

Design; Human Factors.

\section{INTRODUCTION}

Creative professionals often work with paper in the early stages of a creative process, partly for its availability, freedom and lack of constraints [6]. However, this often leads to a problem when the user shifts to a more constrained computer-based tool to implement these ideas, with an abrupt shift in format and interface. Interactive paper technology creates new possibilities for helping such users transition between paper and computer-based data. Unfortunately, our experience designing for desktop computers is not necessarily appropriate in this context. We need a fresh perspective on the design of data components and the interaction techniques and tools used to manipulate them.

J. Garcia, T. Tsandilas, C. Agon, C., and W. E. Mackay. Interactive Paper Substrates to Support Musical Creation. In CHI '12: Proceedings of the SIGCHI Conference on Human Factors in Computing Systems, 4 pages, to appear, ACM 2012.

This is the author's version of the work. It is posted here by permission of ACM for your personal use. Not for redistribution. The definitive version will be published in CHI'12, May 5--10, 2012, Austin, Texas, USA.
Researchers have explored paper-based solutions for a variety of professional users including biologists [9] and music composers [8]. One of the arguments in favor of interactive paper technology is its link between paper and computer, ideally preserving the advantages of each. However, interpreting the handwriting can be a difficult technical challenge. Some researchers address this by restricting the type of gestures, e.g., ModelCraft [7] interprets editing marks in the context of a physical 3D model. Musink [8] goes further by letting users create their own notations and modify those notations over time.

Here, we take another approach. We create paper 'substrates' that accept specialized forms of data which they know how to interpret and process. Classical musicians already work with a highly evolved pre-defined substrate, the musical score, on which time signatures, keys, musical notes, and other marks appear [3]. Contemporary music composers are more idiosyncratic, often creating a new form of musical annotation for each piece. They generate complex, highly individual data and then explore it, creating alternative views, evaluating various solutions and producing new results. Not only do they customize scores and create their own notations [8], they often create entirely new structures upon which to explore musical ideas. Coughlan [1] argues that creative tools should support structural interaction, letting users create the structure in which a creative outcome can be produced. We take this one step further, providing users with personalizable paper substrates that they can customize for the problem at hand.

\section{PAPER SUBSTRATES: DESIGN CONCEPT}

Paper affords expressive ways for exploring data that traditional input devices and interfaces cannot easily support. In a previous study [2], for example, we observed a composer exploring variations of synthetic sounds by superimposing control curves on paper and incrementally evaluating the intermediate results. In order to support such tasks, the paper interface must be equipped with elements that guide the entry and editing of data, automate their synchronization with online representations, and simplify future reuse.

Paper substrates are interactive paper components for working with such data. Each substrate is specialized to handle a certain type of data, which can either be printed or handwritten. Substrates are views of digital representations of data, and are therefore logically connected to computer applications. This means that any data manipulation that takes 
place on a substrate's surface also affects its digital representation. A well-designed substrate respects the affordances of pen and paper: it guides and constrains entry of handwritten data, aids recognition, and offers useful information about its roles and functions. Substrates can be printed as background on a primary sheet of paper or as mobile elements that can be positioned anywhere on the page or other surface in the working environment.

Specialized substrates can be linked together to support more complex data operations and communicate their data and state to each other. As with paper flight strips [5], logical groupings can be defined by means of the spatial positioning of fixed or moveable substrates. Users can also create explicit connections by drawing links between neighboring substrates, which resembles the stitching mechanism Liao et al. [4] proposed for attaching sticky notes to pages. Finally, substrates can be transparent and superimposed to produce physical layers of data and data operations.

\section{PARTICIPATORY DESIGN}

We conducted a series of participatory design sessions with four composers and a musical assistant. The participatory design process allowed us to assess and explore the potential of paper substrates through real-world scenarios that involved manipulating diverse and complex musical data.

Participants. Two composers were graduate composition students in a computer-assisted composition class, another studied at a national music academy and the fourth conducted research on computer-assisted composition. The musical assistant is a music-literate programmer who helped composers produce the electronic parts of their pieces. We refer to them as: $\mathrm{MB}, \mathrm{MGV}, \mathrm{RD}, \mathrm{MK}$ and GB. All were men, aged 25 to 41 .

Design sessions. After a brief introduction about interactive paper technology and our design concept, we encouraged participants to identify scenarios from their previous work for which interactive paper might be useful. Participants described their work process and sketched solutions on paper. We then worked together on the design of an interactive interface for a scenario of their choice. At the end of each session, we created a five-minute video prototype that shows the participant using the interface with his own data. Each session lasted 60-100 minutes.

\section{RESULTS}

Although the participants proposed a number of design ideas, we focused on five distinct scenarios:

- Creating a musical sequence and its orchestration with OpenMusic, a music-programming tool (MB).

- Exploring alternative orchestrations produced by Orchidée, an automatic orchestration tool (MGV).

- Synchronizing a recorded violin part with the original score by using Ableton Live, a music sequencer (RD).

- Creating a small musical piece with PWGL, a musicprogramming tool (MK).
- Generating "sound textures" with cataRT, a sound synthesis tool, by drawing 2D trajectories (GB).

These scenarios helped us to explore the design requirements for paper substrates and how they can support manipulation of a rich and diverse set of data representations.

\section{Substrates as Data Containers}

All participants deal with complex multidimensional data. $\mathrm{MB}$, for example, had to define pitches, durations, intensities, rhythms, instruments, spatialization and other effects for his musical objects. RD used control curves for amplitude, pitches and time onsets. MGV controlled several mathematical variables to generate a set of orchestration solutions, while GB controlled pairs of 20 independent descriptors in space and time.

To represent and interact with such data, participants proposed a number of substrates: containers for musical notation, containers for curves and sound waves, lists of composition rules, lists of parameterized filters, visualizations of points representing alternative solutions, timelines for defining the sequence of modular musical objects, and labels with textual or graphical descriptions. The data in some components were generated on the computer and then printed on paper using either an identical representation or more commonly, a modified representation adapted for paper use. Other components defined specialized templates for entering data with the pen. For example, although musical notation was important for all four composers, each composer designed his own personal musical substrate. RD created zones of printed score segments whose actual content had been generated on the computer. MGV created a specialized area with empty musical staffs (Figure 1).

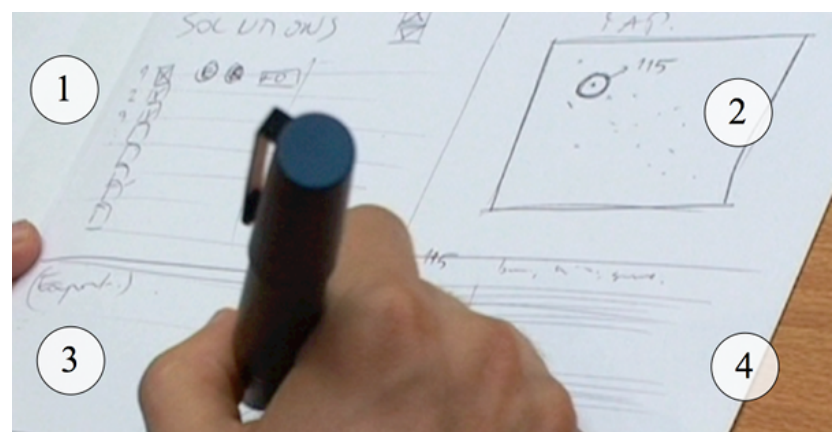

Figure 1: Various data containers in MGV's prototype. 1: Selection of active instruments with checkboxes.

2: Selection of solutions by circling parameterized data points. 3: Export button to save a particular state of the solution. 4: Free area for classical music notations.

The handwritten musical content in this area was stored but not translated by the computer and served as a musical annotation on a selected orchestration solution. MK created both areas with printed musical content, which represented composition rules or predefined music sequences, and areas with empty staffs dedicated to the entry of note intervals. Finally, MB divided the entry of the variables from a musical segment into three interconnected paper substrate strips 
that enter: (1) pitches as notes; (2) rhythms as gestures; and (3) spatialization definitions as points. This strategy is particularly interesting because it simplifies the recognition of handwritten musical notation and promotes reuse.

\section{Connecting and Positioning Substrates}

In all scenarios, several data components acted as layers that control different parameters of the same musical object. The connection between the layers was either fixed or could change dynamically during the task. For example, in RD's prototype (Figure 2), all the data components that control a musical object are layered vertically on the same page. RD explained that this layout makes "easier to organize [his work] on paper and helps him structure the data".

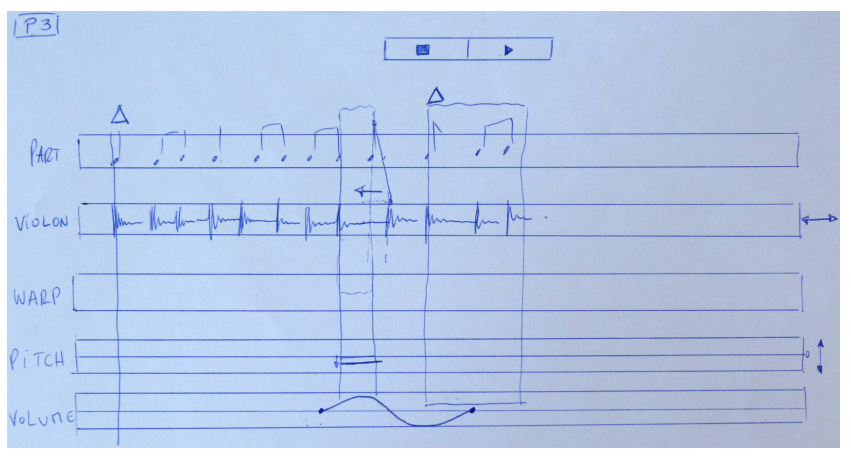

Figure 2: RD's prototype with time-aligned layers for sound editing, including symbolic representations of a violin recording with modification curves above.

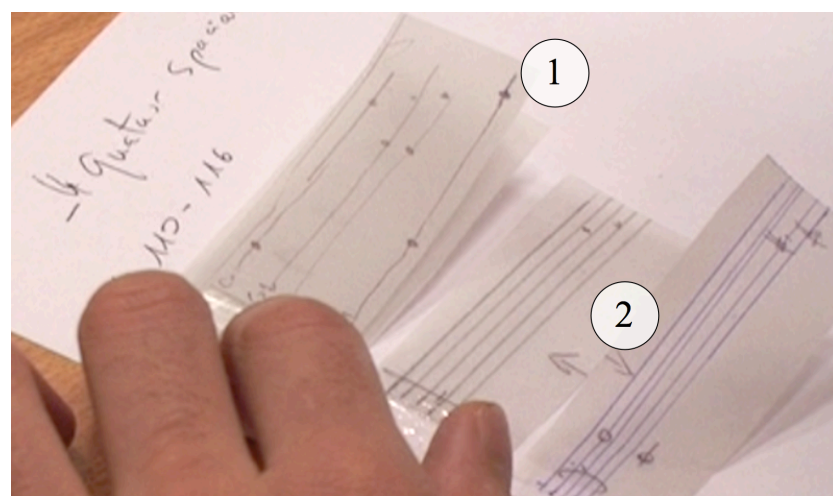

Figure 3: MB's prototype for linking musical objects.

1: Transparent elements define rhythm and spatialization. 2: Arrows link two pitch input elements. Connected elements (linked or stacked) are unique musical objects but share time.

In contrast, $\mathrm{MB}$ used small movable strips of paper that could be placed anywhere on the page. In order to logically associate two strips together, he aligned them physically and used the pen to draw a link. MB also used translucent strips to create physical layers of data (Figure 3 ). The decision about whether to use fixed or moveable substrates varied across participants. RD and MGV worked with preprinted data components. The other three participants preferred using movable paper components that could be freely positioned, mixed and reused. The support for movable components was particularly important for MB. He com- mented that he could never make prior decisions about the structure of his work on paper.

\section{Modules}

MK introduced the concept of paper modules (Figure 4), borrowing the modular structure of the composition tool that he used. Modules act as programmable entities that can be reused in different sequences to produce different variations. In MK's workspace, paper modules are labeled data components that represent composition rules, functions or musical elements. MK used labels to apply rules or rearrange musical elements along timelines.

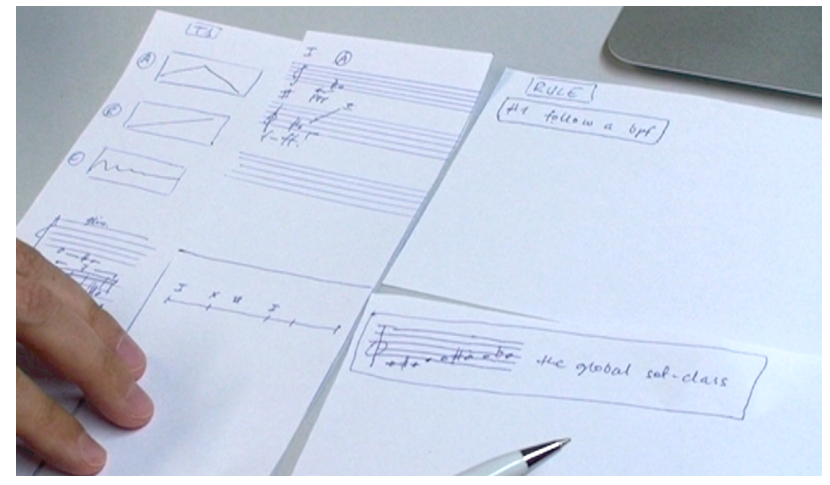

Figure 4: MK's prototype comprises distinct paper modules with specific functions: areas for curves, predefined music sequences, note input, lists of rules, and a timeline.

\section{Archiving and Reuse}

Some composers expressed the need for keeping a history of their completed work. They explained that they regularly return to their previous pieces to find interesting material. MK told us that even famous composers like Stravinsky reused their older material in new pieces. To support future reuse, $\mathrm{MB}, \mathrm{MGV}$ and $\mathrm{MK}$ added identifiers such as textual descriptions, numbers, and graphical elements on paper. Finally, MGV came up with the idea of project folders that contain alternative solutions (or variations) of a given task. Each time a new solution is produced on a page, it is labeled with the project identifier and stored in the folder.

\section{INTERACTIVE PROTOTYPE}

Inspired by the design ideas of our participants, we implemented an interactive, tangible prototype for editing musical sequences on paper (Figure 5). The prototype demonstrates the technical feasibility of the concept of paper substrates and concretizes it via simple tasks accessible to both expert musicians and non-experts.

We used Anoto paper technology and ADP-301 digital pens. The pens communicate in real time with a computer through a Bluetooth connection. A Java application registers and interprets events generated as the user interacts with the substrates and provides direct audio and visual feedback through a Max/MSP patch. The communication between the Java application and Max/MSP is based on OSC (http://opensoundcontrol.org/). The prototype allows the user to import a musical sequence from music software 
and print a simplified representation of this sequence on paper as an interactive substrate. The user can also create and print instances of two other specialized types of substrates: a graph component, and a playbar component. When connected with a sequence component, the graph and playbar components obtain the musical sequence's timeline. At run time, users can modify onsets and durations of printed notes by drawing line segments over their rectangular representations. They can draw control curves in the graph component and then change them incrementally. Finally, they can use the playbar component to select slices in the timeline and replay the corresponding sequence. Each paper substrate is associated with a different musical object in a Max/MSP patch. The state of the online object changes in real time while the user interacts with the data on paper. We have experimented with both regular and translucent paper and support both fixed paper substrates and moveable ones. The latter can be dynamically linked together by drawing simple strokes between their margins. Users can create these links to position or reposition graph and playbar components along the timeline of a musical sequence. Links can later be removed by crossing the trace of the link with a new mark.

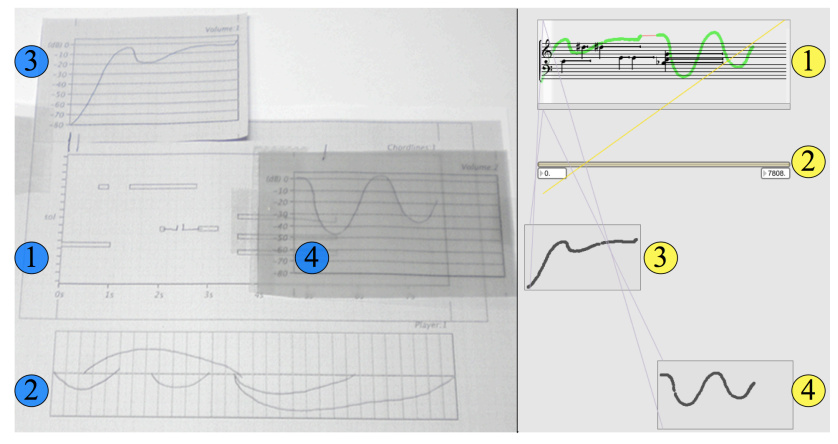

Figure 5: Paper substrates for editing a musical sequence. Left: Printed interface with physical data (blue). Right: Max/MSP interface with digital data (yellow).

Figure 5 shows how several graph (substrates 3 and 4) and player components (substrate 2) can be connected to the same musical sequence (substrate 1). The Max/MSP patch (right) visualizes the structure of the substrates linked to the active sequence and updates the edited musical score with real time data from the pen. Graphical data sent by graph substrates are automatically concatenated and applied to the musical object, either to control its volume or of a background sound effect. Users may draw arcs over the playbar substrate to make selections along its timeline. They can then play the selections and listen to the result by tapping the pen over the arcs.

\section{CONCLUSION AND FUTURE DIRECTIONS}

Our goal is to design novel interactive paper interfaces that support the creative process. We explored the potential of a new concept, paper substrates, in five participatory design sessions. Substrates are paper components with strong data types that support advanced interaction depending upon context. This concept fits well with composers' existing practices, since they are already familiar with the concept of substrates as musical scores and symbolic notation. The participatory design sessions demonstrated its power, as participants created, manipulated and combined layers of data, rearranging them in time and space as an integral part of the creative process. Moreover, the substrates approach fully supports an iterative process in which templates can evolve and be reused, resulting in highly personal and powerful interfaces.

We observed that paper substrates could take on different roles, serving as data containers, data filters, and selectors. The design sessions resulted in several pen interactions and tangible manipulations of paper components to support these roles: drawing and modifying specialized data over formatted paper, exploring variations by superimposing handwritten data, defining programmable modules, aligning movable substrates, linking them together, overlaying them, and archiving them into physical folders.

Our future goal is to develop interfaces that support the substrates proposed by our participants. Finally, we are planning to design tools that help composers customize the structure of their paper interfaces and create their own specialized substrates.

\section{REFERENCES}

1. Coughlan, T. and Johnson, P. Understanding productive, structural and longitudinal interactions in the design of tools for creative activities. In Proc. $C \& C^{\prime} 09$ (2009), ACM, 155-164.

2. Garcia, J., Tsandilas, T., Agon, C. and Mackay, W. InkSplorer: Exploring Musical Ideas on Paper and Computer. In Proc. NIME'11 (2011), 361-366.

3. Gardener, R. Music Notation, a Manual of Modern Practice. London: V. Gollancz, 1982.

4. Liao, C., Guimbretière, F., Hinckley, K. and Hollan, J. Papiercraft: A gesture-based command system for interactive paper. In TOCHI, 14(4), 2008, ACM, 1-27.

5. Mackay, W.E. Is paper safer? The role of paper flight strips in air traffic control. In TOCHI, 6(4), 1999, ACM, 311-340.

6. Sellen, A.J. and Harper, R.H.R. The Myth of the Paperless Office. (2003). MIT Press.

7. Song, H., Guimbretière, F., Hu, C. and Lipson, H. ModelCraft: capturing freehand annotations and edits on physical 3D models. In Proc. UIST'06 (2006), ACM, 13-22.

8. Tsandilas, T., Letondal, C. and Mackay, W.E. Musink: composing music through augmented drawing. In Proc. CHI'09 (2009), ACM, 819-828.

9. Yeh, R., Liao, C., Klemmer, S., Guimbretière, F., Lee, B., Kakaradov, B., Stamberger, J. and Paepcke, A. ButterflyNet: a mobile capture and access system for field biology research. In Proc. CHI'06 (2006), ACM, 571580. 